\title{
Laboratory rearing of Anopheles arabiensis: impact on genetic variability and implications for Sterile Insect Technique (SIT) based mosquito control in northern Sudan
}

\author{
Rasha Siddig Azrag ${ }^{1,2^{*}} \mathbb{D}$, Kamal Ibrahim $^{3}$, Colin Malcolm ${ }^{4}$, Elamin El Rayah² and Badria El-Sayed
}

\begin{abstract}
Background: Mosquito colony populations often show significant changes in their population genetic make-up compared to the field populations that were used as founding source. Most of the changes that have been reported are indicators of depletion in the overall genetic diversity of the colony populations. The Sterile Insect Techniques programme of mosquito control that is underway in Northern Sudan uses sterilized males produced from a laboratory-maintained colony population. The genetic diversity of an advanced generation of this colony population was quantitatively assessed and compared to the field population from which the colony was derived.
\end{abstract}

Methods: Anopheles arabiensis mosquito samples from the 13th generation of the colony, and from the locality that was the source of the first generation of the colony, were genotyped at 11 microsatellite loci distributed throughout the species' genome. Standard population genetic analyses were carried out to quantify and compare their population genetic make-up and diversities.

Results: The colony samples showed significant reduction in the total number of alleles, the numbers of rare and private alleles, and the fractions of heterozygote individuals at all the loci. The pattern of change is consistent with the expected effect of the use of a small number of mosquitoes when the colony was established. Departure from Hardy-Weinberg equilibrium in the direction of homozygote excess was observed at some loci and attributed to the presence of null-alleles.

Conclusions: This study highlights the need for broad sampling when initiating colony populations and for ongoing assessment of the population genetic make-up of colony populations. Previous assessments of survivorship, dispersive behaviour and swarm formation indicate that the inbreeding and reduced genetic variability reported in this study may not have had direct fitness consequences yet. However, noting the lessons learned in other SIT programmes about the impact of colonization on male sexual behaviour and longevity, as well as other inbreeding related adverse effects, a systematic investigation of these potential effects is recommended because they have direct impact on the ultimate success of the programme.

Keywords: Anopheles arabiensis, Mosquitoes, Colonization, Genetic variability, Microsatellites, Sterile insect technique

\section{Background}

Insect species are reared in laboratories to produce colony populations from which a steady supply of the desired

\footnotetext{
*Correspondence: razrag@hotmail.com

${ }^{1}$ Tropical Medicine Research Institute, National Centre for Research, Khartoum, Sudan

Full list of author information is available at the end of the article
}

life-stage can be obtained for research as well as for biological control purposes. The laboratory colonies are either representative of the field populations from which they originate or serve as a stable standard to which other populations may be compared. Many studies have examined the genetic consequences of artificial rearing when compared to wild populations; some with focus on the 
implications on using the laboratory animals in pathogen transmission studies. These studies have revealed that the genetic make-up of colony populations often shows significant departures from the originally sampled field populations. Common features of the departures include: reduced number of alleles, divergence in allele frequency, presence of new alleles, reduced heterozygosity, fewer multi-locus genotypes, fewer rare alleles, and, reduced number of private alleles. Most of the changes that have been reported are indicators of depletion in the overall genetic diversity [1-4]. The consequences of inbreeding on reproductive traits of laboratory strains overtime has also been examined [5].

Some of the common genetic changes that accompany laboratory rearing of insect colonies are not universal. A substantially lower rate of fixation at several enzyme loci in inbred stocks of the tree-hole mosquito, Aedes triseriatus, was reported and it was concluded that lethality of homozygous recessives at these loci accounts for the elevated heterozygosity [6]. Similarly, [7] were able to demonstrate that inbred lines of the Anopheles gambiae sensu stricto retained discrete genomic blocks that maintained high heterozygosity due to polymorphic chromosomal inversions. Broadly speaking, comparisons of the genetics of colony populations and field populations is important particularly in genetic control trials because population genetic make-up can affect physiological and behavioural fitness traits, and hence the efficacy of the genetic control methods [8-10]. The fitness of Anopheles arabiensis male populations in SIT trials in northern and south Africa, showed that prolonged colonization, irradiation, and transportation do not impede mating vigour and competitiveness of male mosquitoes [11-14]. Colonization of An. arabiensis under semi-field conditions was also shown to be associated with the retention of a higher degree of genetic diversity, reduced inbreeding and greater phenotypic similarity to the founding wild population than observed in laboratory-based small cage colonies [15].

This study assessed the allelic and genotypic diversity of the An. arabiensis Patten, 1905, colonies that are maintained at the Tropical Medicine Research Institute (TMRI) in Khartoum, Sudan. The aim was to explore the implications of any changes in the genetic makeup of the colony population to the ongoing trials of Sterile Insect Technique (SIT) based mosquito control in northern Sudan.

\section{Methods}

\section{Source population}

The mosquito species An. arabiensis is the only member of the Anopheles gambiae species complex that is found in northern Sudan and it is the major malaria vector in the region. Mosquito larvae were collected from the Kabtoad area of Dongola town, from water pools along the banks of River Nile [12]. Larvae were collected from different habitats including leaking water pipes, grassy river banks and stagnant water in potholes using the classical dipping method [16]. The larvae were cleaned from predators and other Culex mosquito larvae, pooled together and transported in plastic containers to the insectary at the TMRI in Khartoum where they were reared. Sample sizes from each habitat type varied; when pooled, there were sufficient larvae to stock 3 white dishes with a minimum of 300 larvae each as described below.

\section{Laboratory rearing}

The TMRI insectary, established in 2002, maintains a controlled environment of between 17 and $20{ }^{\circ} \mathrm{C}$ temperature and relative humidity of $80 \%$. The mosquito larvae were placed in large white dishes $(30 \mathrm{~cm} \times 20 \mathrm{~cm})$, kept under light for $10 \mathrm{~h}$ per day and fed commercial baby food. The larvae were kept in the water from their larval habitat; most pupated without adding food. Pupae were collected using a dropper and transferred into $30 \mathrm{~cm} \times 30 \mathrm{~cm}$ cages. Emergent adults were fed on glucose sugar (5\%). Subsequently, adult mosquitoes were fed human or rabbit blood at sunset and at night. Successive generations were reared in this manner. The main food for larvae for subsequent generations was baby food (Nestle, Cerelac). The pupae of the first generation of eggs reared in the laboratory were transferred into cages labelled G1 and reared under the same laboratory conditions. Single adult flies were collected from these cages using an aspirator, replaced in $-20^{\circ} \mathrm{C}$ for 2 min to knock down the mosquito then quickly single mosquitoes were placed in a labelled cryotube and preserved in $90 \%$ ethanol at $-20{ }^{\circ} \mathrm{C}$ for microsatellites analysis. All larval dishes were covered with fine mesh. The average duration of each colony generation was around 3 weeks. However, the early generations, especially G1-G3 required longer time due delayed egg hatching or restocking of adult samples. This extended the duration of the colony establishment phase.

\section{DNA extraction}

DNA extraction from individual insects was carried out using a potassium acetate lysis buffer followed by alcohol precipitation as described in [17]. The extracted DNA was dissolved in $100 \mu \mathrm{l}$ of T.E., $\mathrm{pH}$ 8.0. and stored at $-20{ }^{\circ} \mathrm{C}$ until used in PCR amplification.

\section{Species confirmation}

PCR using species-specific primer pairs was used to confirm identification. The universal mosquito primer UN-5'GTGTGCCCCTTCCTCGATGT3' was used as a 
forward primer, paired with the Anopheles gambiae specific reverse primer GA-5'CTGGTTTGGTCGGC ACGTTT3' or the An. arabiensis specific reverse primer: AR-5'AAGTGTCCTTCTCCATCCTA3' according to [18]. The PCR cocktail contained $1.2 \mu \mathrm{M}$ of each of the four dNTPs, 0.5 units Tag polymerase, $0.5 \mu \mathrm{M}$ each forward and reverse primers made up to a total volume of $25 \mu \mathrm{l}$ with PCR buffer containing $1 \mathrm{mM} \mathrm{MgCl}$. The PCR was carried out in an ABI 97000 Gene Amp thermal cycler as follows. Initial denaturing for $4 \mathrm{~min}$ at $94{ }^{\circ} \mathrm{C}$ followed by 30 cycles of denaturation at $94{ }^{\circ} \mathrm{C}$ for $30 \mathrm{~s}$, annealing at $50{ }^{\circ} \mathrm{C}$ for $30 \mathrm{~s}$ and extension at $72{ }^{\circ} \mathrm{C}$ for $30 \mathrm{~s}$, followed by a final extension for $5 \mathrm{~min}$ at $72^{\circ} \mathrm{C}$.

\section{Microsatellite analysis}

Eleven primer pairs that amplify microsatellite loci located on autosomal chromosomes 2 and 3, and the X chromosome as described in [19] and [20] were used. The primer sequences, their annealing temperatures, previously reported expected modal allele size and the repeat motif are shown in Table 1.

Microsatellite genotyping was carried out according to [21]. The forward primer of each locus was $5^{\prime}$ labelled with a FAM, HEX or NED fluorescent tag, followed by PCR amplification as shown in the species confirmation protocol above, except for an annealing temperature of $55^{\circ} \mathrm{C}$. PCR products were scanned in an ABI PRISM 3700 sequencer (Applied Biosystem) following manufacturer's protocols. The Genotyper DNA Fragment Analysis Software (Applied Biosystems) was used to call allele sizes.

\section{Data analysis}

The software package Tandem [22] was used for binning allele sizes. The output file from Tandem was used as input file in Convert [23] to obtain data files for use in the population genetic software packages Web Genepop [24] and Arlequin [25]. The latter were used to obtain allele and genotype frequencies and to test for deviation from Hardy-Weinberg equilibrium (HWE). The AmCharts JavaScript library was used to draw nested pie charts depicting the frequency distributions of the alleles at the nine linkage group 2 and 3 loci.

\section{Results}

\section{Allele sizes}

A total of 58 An. arabiensis mosquitoes collected from Dongola area, henceforth referred to as field population, were compared to 51 samples from generation 13 of the An. arabiensis colony maintained at the TMRI insectary, referred to as colony population. DNA from each sample was PCR amplified using the species specific primers to confirm species identification and subsequently genotyped at 11 microsatellite loci, nine of which have been mapped to linkage groups (chromosomes) 2 and 3 while two are on the X chromosome [20]. Binned allele sizes at all the loci varied by multiples of the respective repeat motifs shown in Table 1.

\section{Allele frequencies and rare alleles}

Figure 1 compares allele frequencies at the 11 microsatellite loci in the field and colony populations. The donut plots for all but one of the 11 loci clearly show a dramatic reduction in the number of alleles in the colony population (left half) compared to the source population from Dongola (right half). Only one locus, Ag3H93, retained the same number of alleles in both populations (Table 2). We note that in all the loci where allele numbers declined, the commonest allele retained in the 13th generation of colony population is either the commonest or one of the common alleles in the field population. The average number of alleles per locus in the colony

Table 1 The microsatellites loci used in the study $[9,10]$

\begin{tabular}{|c|c|c|c|c|}
\hline Locus & Foreword primer & RM & Reverse primer & $A_{s}$ \\
\hline $\mathrm{AgXH7}$ & CACGATGGTTTTCGGTGTGG & (GT)8 & ATTTGAGCTCTCCCGGGTG & 99 \\
\hline $\mathrm{AgXH180}$ & GTATGTTGTGATCTCCTGCC & (GT)10 & AAAACGAGCCACCACCAGAG & 72 \\
\hline $\mathrm{Ag} 2 \mathrm{H} 175$ & AGGAGCTGCATAATTCACGC & $(\mathrm{CA}) 8$ & AGAAGCATTGCCCGCATTCC & 97 \\
\hline $\mathrm{Ag} 2 \mathrm{H} 1010$ & GCGTATGTCAATGGCGAGAA & (GATA)6 & CGCTGGAAATTGTCACACC & 117 \\
\hline $\mathrm{Ag} 2 \mathrm{H} 46$ & CGCCCATAGACAACGAAAGG & (GT)8 & TGTACAGCTGCAGAACGAGC & 138 \\
\hline $\mathrm{Ag} 2 \mathrm{H} 26$ & GGTTCCTGTTACTTCCTGCC & (GT)8 & CCGGCAACACAAACAATCGG & 154 \\
\hline $\mathrm{Ag} 2 \mathrm{H} 143$ & CGTACGAGTGAGTGAGTTGG & $(\mathrm{TC}) 9$ & CAAAAATAGCATCACGGCCG & 160 \\
\hline $\mathrm{Ag} 3 \mathrm{H} 249$ & ATGTTCCGCACTTCCGACAC & (GT)15 & GCGAGCTACAACAATGGAGC & 129 \\
\hline $\mathrm{Ag} 3 \mathrm{H} 88$ & TGCGGCGGTAAAGCATCAAC & (GT)9 & CCGGTAACACTGCGCCGAC & 176 \\
\hline $\mathrm{Ag} 3 \mathrm{H} 93$ & 5' 8TCCCCAGCTCACCCTTCAAG3' & (GT) $4+7$ & 3'GGTTGCATGTTTGGATAGCG5' & 209 \\
\hline $33 \mathrm{C} 1$ & 5'8TTGCGCAACAAAAGCCCACG3' & $(A G C) 6$ & 3'ATGAAACACCACGCTCTCGG5' & 159 \\
\hline
\end{tabular}

$R M$ repeat motif, $A_{T}$ annealing temperature, $A_{S}$ expected allele size in bp 


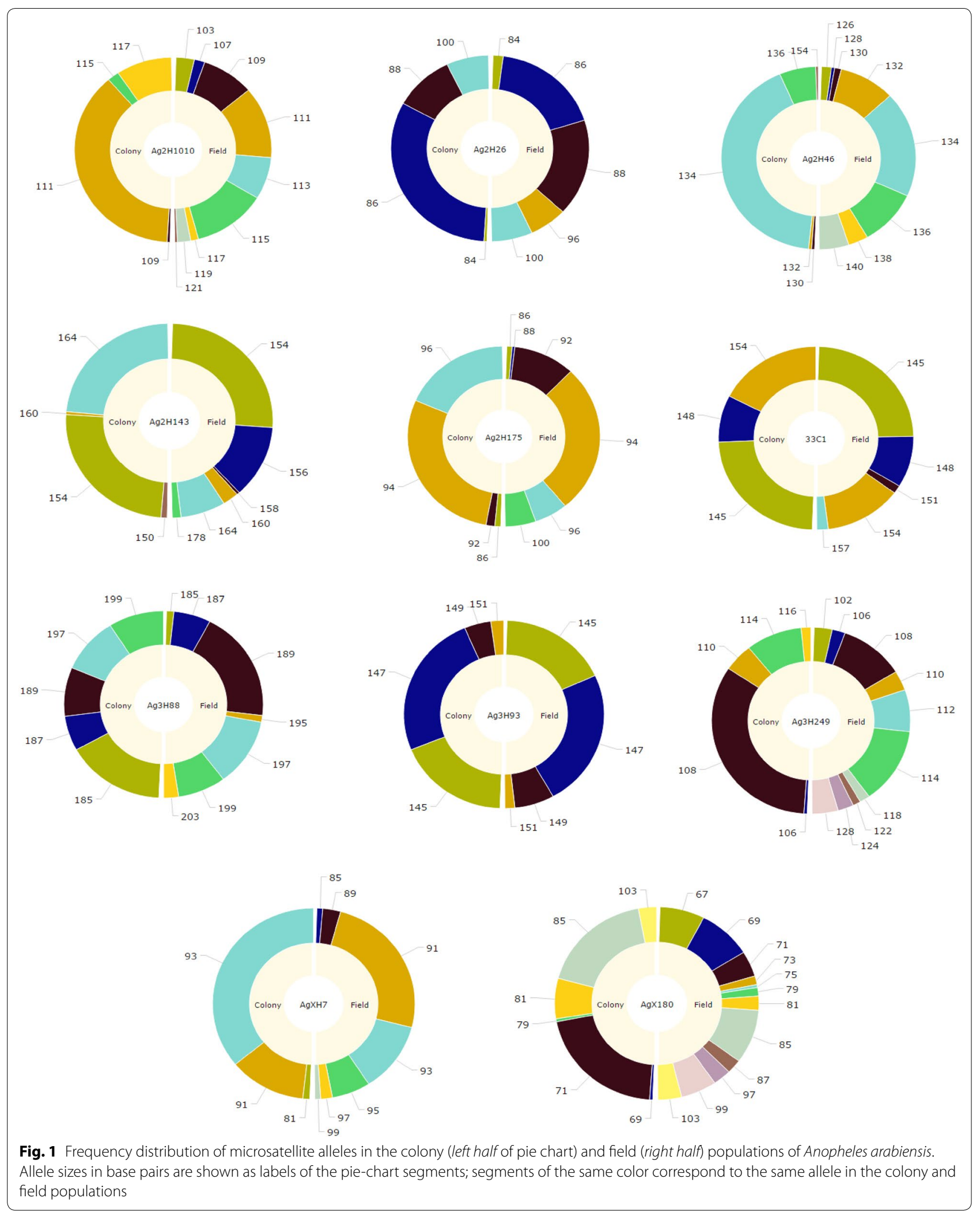




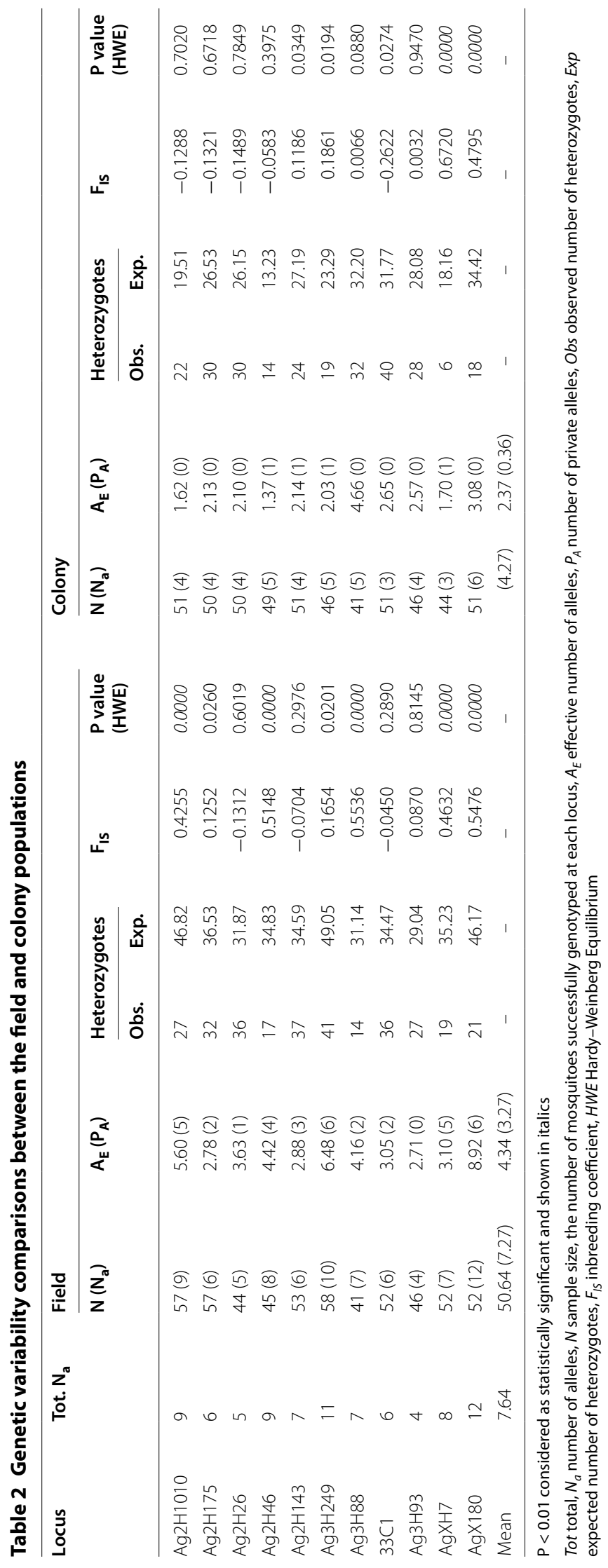


population (4.27) was reduced by $40 \%$ compared to the field population (7.27). These are the expected trends under a scenario of divergence from the source that is due to founder effect coupled with subsequent drift during the 13 generations of colony maintenance. All tests of genic differentiation, i.e. test of the statistical significance of the allele frequency divergence between the two populations, were highly significant $(\mathrm{p}<0.001)$. The number of rare alleles, defined as alleles with frequencies of less than $5 \%$ in either population, is another contrasting feature between the wild population and the laboratory colony. There were a total of 29 rare alleles in the former, an average of 2.64 per locus. The corresponding estimates for the colony population were 16 alleles, an average of 1.45 alleles per locus.

\section{Private alleles and effective number of alleles}

A private allele is an allele present in one, but not the other of the two populations that were compared. The total number of private alleles combined across all loci was 40 , of which 36 were private to the field population. Averaged across all 11 loci, the number of private alleles in the field population was almost ten times as many as that in the colony population, 3.27 vs 0.36 private alleles per locus (Table 2). This is further evidence of the significant reduction in genetic diversity experienced by the colony population at founding and during the 13 generations of insectary propagation.

The effective number of alleles $\left(A_{E}\right)$ is a measure of allele diversity, which is used when comparing populations in which the number of alleles and their frequency distributions differ drastically. It is defined as the number of equally frequent alleles it would take to achieve a given gene diversity, represented by the expected heterozygosity $\left(\mathrm{H}_{\mathrm{e}}\right)$ of a population. It is computed as $\mathrm{A}_{\mathrm{E}}=(1-(1 /$ $\mathrm{H}_{\mathrm{e}}$ ). Again, a consistent trend of reduction in the effective number of alleles at all but one locus is evident in the colony population compared to the field population (Table 2). Average $A_{E}$ in the colony population has been reduced by $45 \%$. This is comparable to the reduction in the raw allele count; it appears that the difference in the number of alleles per locus contributes more to the divergence between the two populations than does the difference in allele frequencies.

\section{Genotype frequencies, Hardy-Weinberg equilibrium}

The average number of heterozygotes per locus is higher in the field population reflecting the higher number of alleles per locus. Furthermore, in both the field and the colony populations, where statistically significant, the deviations from HWE expectations were always in the direction of homozygote excess (Table 2). The colony samples were at HWE at all the autosomal loci; the field population showed significant homozygote excess in three of them as did both populations at the two X-linked loci.

\section{Discussion}

This study confirms that microsatellite allelic and genotypic diversities have declined considerably in the TMRI colony of mosquitoes compared to the field population from which it was established. Comparable loss of diversity has been reported in Mali by Norris et al. [3] at nine microsatellite loci in two colonies of Anopheles gambiae compared to a field population. Baeshen et al. [5] also reported significant reduction in genetic diversity and increase in homozygosity in a similar study involving Aedes triseriatus populations. The mostly likely causes of the observed changes are discussed below, followed by their implications to the sterile insect techniques (SIT) based mosquito control programme underway in Northern Sudan that uses the TMRI population as source of sterile male mosquitoes.

The contrasting features of the genetic diversities of the wild and colony populations, taken individually or combined, could be due to the founder effect. When a new population is founded from a small, random sub-sample of a genetically diverse population the common alleles in the source population are much more likely to be sampled than the rarer alleles, and, there is bound to be a reduction in diversity. This is borne by the data in that the common alleles retained in the colony population are also common in the field population (Fig. 1). The reduction in the total number of alleles, the private alleles and the rare alleles is also most likely to be the outcome of founder effect. Drift during the 13 generations of propagation in the laboratory could have contributed, but the size of the colony population in successive generations, which was significantly bigger than the typical population size at a breeding site, was large enough to minimize this. Thus, it is concluded that limited sampling during colony establishment contributed most to the contrasting patterns of genic diversity of the field and colony populations. The fact that Hardy-Weinberg genotypic proportions were confirmed at all the autosomal loci in the colony population implies that the reduction in average heterozygosity compared to the field population is due to the reduction in the number of alleles. On the other hand, the homozygote excess at three autosomal loci in the field population and the two X-linked loci in both populations is attributable to the presence of null-alleles. Other potential evolutionary causes of excess homozygosity at loci that are presumed neutral are expected to affect most if not all 11 loci. 
A number of recent studies comparing colonization of An. arabiensis under semi-field conditions to laboratory-rearing in small cages have reported that the former retained a higher level of genetic diversity, showed less inbreeding and were phenotypically more similar to the founding wild population $[5,15,26]$. The cage-reared TMRI colony population falls in the latter category. Reduction in genic diversity and increase in homozygosity could depress fitness as a result of the expression of deleterious recessive alleles combined with the loss of heterozygote advantage. This trend has been confirmed using pedigree-based breeding experiments, which showed a decline in fitness-associated traits in individuals with high inbreeding coefficients [27-29]. However, the generality of the heterozygosity-fitness correlations in natural populations is difficult to assess, because most studies that have evaluated the relationship in the wild are either experimental or performed on smaller isolated populations [30-32].

All novel insect control strategies involving mass release must address the issue of the evolutionary trajectory of genetic diversity in the released control agents relative to the source. This study has not confirmed or excluded the possibility that the elevated inbreeding in the colony population compared to the source population has led to loss of mating vigour in the sterile males, or any other change in their reproductive characteristics, or the evolution of premating isolation mechanisms. However, relevant insight has been gained from other studies focusing on the impact and consequences of colonization, mass rearing and loss of diversity within the SIT trial programme in northern Sudan. Excoffier and Lischer [25], who studied the capacity of released sterile An. arabiensis males to survive, disperse and participate in swarms occurring at varying distances from the release site, showed that sterile An. arabiensis males released into the field were able to find and participate in existing swarms. Helinski et al. [11] and Hassan et al. [12] reported no major obstacles associated with the small-scale irradiation and transportation of An. arabiensis males in the current SIT setting and laboratory-reared and irradiated An. arabiensis males from sixty generations were able to inseminate wild females at rates comparable to wild males. Mating competitiveness experiments showed that irradiated male mosquitoes are fertile as wild counterparts under semi-field conditions. However, they were not as competitive under laboratory conditions [14].

\section{Conclusions}

This study highlights the need for broad sampling when initiating mosquito colony populations. Ongoing assessment of the population genetic make-up of colony populations at regular intervals is also recommended. The SIT programme in Northern Sudan has an active research programme that has looked into survivorship, dispersive behaviour and swarm formation in the colony population we studied. These studies have indicated that the inbreeding and reduced genetic variability we report may not have had direct impact on the traits that were investigated. However, we note the lessons learned in other SIT programmes about the impact of the population genetic makeup of release populations on male sexual behaviour [33], on the relative longevity of sterile males [34], and, on the extent to which inbreeding related adverse effects may be mitigated by occasional outbreeding of colony populations with wild caught mosquitoes [5]. A systematic investigation of these potential consequences of loss of genetic diversity is recommended because they have direct and significant impact on the ultimate success of the SIT programme in the Sudan.

\section{Abbreviations}

IAEA: International Atomic Energy Agency; SIT: Sterile Insect Technique; TMRI: Tropical Medicine Research Institute-Khartoum, Sudan.

\section{Authors' contributions}

RA gathered the data and carried out the experiments as part of her Ph. D. dissertation. RA and KMI contributed equally to data analyses and drafting of the paper. All authors read and approved the final manuscript.

\section{Author details \\ ${ }^{1}$ Tropical Medicine Research Institute, National Centre for Research, Khartoum, Sudan. ${ }^{2}$ Department of Zoology, Faculty of Science, University of Khartoum, Khartoum, Sudan. ${ }^{3}$ Department of Zoology, Southern Illinois University Carbondale, Carbondale, IL 62901, USA. ${ }^{4}$ School of Life and Medical Sciences, University of Hertfordshire, Hatfield, UK.}

\section{Acknowledgements}

We would like to thank the staff of the Genome Center Laboratory, School of Medicine and Dentistry, Queen Mary University of London. This manuscript was significantly improved by the suggestions of two anonymous reviewers.

\section{Competing interests}

The authors declare that they have no competing interests.

\section{Availability of data}

The microsatellite genotypic data used in this study are available at the Dryad Digital Data Depository (http://datadryad.org/)

\section{Ethics approval and consent to participate}

This research involves no human subjects but has used human material. As it relates to the latter, we have obtained protocol approval from the Tropical Medicine Research Institute, Khartoum Sudan (number 2/2016), and we have complied with the institutional regulations of the University of Khartoum and the national guidelines in the Sudan.

\section{Funding}

This work received financial and technical support from the International Atomic Energy Agency (IAEA) and the Tropical Medicine Research Institute, National Center for Research, Sudan.

Received: 24 May 2016 Accepted: 11 August 2016

Published online: 25 August 2016 


\section{References}

1. Munstermann LE. Unexpected genetic consequences of colonization and inbreeding: allozyme tracking in Culicidae (Diptera). Ann Entomo Soc Am. 1994;87:158-64.

2. Mukhopadhyay J, Rangel EF, Ghosh K, Munstermann LE. Patterns of genetic variability in colonized strains of Lutzomyia longipalpis (Diptera: Psychodidae) and its consequences. Am J Trop Med Hyg. 1997;57:216-21.

3. Norris DE, Shurtleff AC, Touré YT, Lanzaro GC. Microsatellite DNA polymorphism and heterozygosity among field and laboratory populations of Anopheles gambiae s.s. (Diptera: Culicidae). J Med Entomol. 2001;38:336-40.

4. Nayar JK, Knight JW, Munstermann LE. Temporal and geographic genetic variation in Culex nigripalpus Theobald (Culicidae: Diptera), a vector of St. Louis encephalitis virus, from Florida. J Med Entomol. 2002;39:854-60.

5. Baeshen R, Ekechukwu NE, Toure M, Paton D, Coulibaly M, Traoré SF, et al. Differential effects of inbreeding and selection on male reproductive phenotype associated with the colonization and laboratory maintenance of Anopheles gambiae. Malar J. 2014;13:9.

6. Matthews TC, Craig GB. Heterozygosity in inbred strains of the tree-hole mosquito Aedes triseriatus. Biochem Genet. 1987:25:647-55.

7. Turissini DA, Gamez S, White BJ. Genome-wide patterns of polymorphism in an inbred line of the African malaria mosquito Anopheles gambiae. Genome Biol Evol. 2014;6:3094-104.

8. Moreira LA, Wang J, Collins FH, Jacobs-Lorena M. Fitness of anopheline mosquitoes expressing transgenes that inhibit Plasmodium development. Genetics. 2004;166:1337-41.

9. Catteruccia F, Godfray HCJ, Crisanti A. Impact of genetic manipulation on the fitness of Anopheles stephensi mosquitoes. Science. 2003;299:1225-7.

10. Benedict MQ, Robinson AS. The first releases of transgenic mosquitoes: an argument for the sterile insect technique. Trends Parasitol. 2003;19(8):349-55.

11. Helinski ME, Hassan MM, El-Motasim WM, Malcolm CA, Knols BG, El-Sayed B. Towards a Sterile Insect Technique field release of Anopheles arabiensis mosquitoes in Sudan: irradiation, transportation, and field cage experimentation. Malar J. 2008;7:65

12. Hassan MM, El-Motasim WM, Ahmed RT, El-Sayed BB. Prolonged colonisation, irradiation, and transportation do not impede mating vigour and competitiveness of male Anopheles arabiensis mosquitoes under semifield conditions in northern Sudan. Malar World J. 2010;1:1-8.

13. Ageep TB, Damiens D, Alsharif B, Ahmed A, Salih EH, Ahmed FT, et al. Participation of irradiated Anopheles arabiensis males in swarms following field release in Sudan. Malar J. 2014;13:484.

14. Munhenga G, Brooke BD, Gilles JRL, Slabbert K, Kemp A, Dandalo LC, et al. Mating competitiveness of sterile genetic sexing strain males (GAMA) under laboratory and semi-field conditions: steps towards the use of the Sterile Insect Technique to control the major malaria vector Anopheles arabiensis in South Africa. Parasit Vectors. 2016;9:122.

15. Ng'habi KR, Lee Y, Knols BGJ, Mwasheshi D, Lanzaro GC, Ferguson HM. Colonization of malaria vectors under semi-field conditions as a strategy for maintaining genetic and phenotypic similarity with wild populations. Malar J. 2015;14:1-11.

16. WHO: Manual on practical entomology in malaria. Part II. methods and techniques. Geneva: World Health Organization; 1975.
17. Miller SA, Dykes DD, Polesky HF. A simple salting out procedure for extracting DNA from human nucleated cells. Nucleic Acids Res. 1988; $16: 1215$

18. Scott JA, Brogdon WG, Collins FH. Identification of single specimens of the Anopheles gambiae complex by the polymerase chain reaction. Am J Trop Med Hyg. 1993;49:520-9.

19. Zheng L, Benedict MQ, Cornel AJ, Collins FH, Kafatos FC. An integrated genetic map of the African human malaria vector mosquito Anopheles gambiae. Genetics. 1996;143:941-52.

20. Lehmann T, Hawley WA, Kamau L, Fontenille D, Simard F, Collins FH. Genetic differentiation of Anopheles gambiae populations from east and west Africa: comparison of microsatellite and allozyme loci. Heredity. 1996;77:192-208.

21. Wang R, Kafatos FC, Zheng L. Microsatellite markers and genotyping procedures for Anopheles gambiae. Parasitol Today. 1999;15(1):33-7.

22. Matschiner M, Salzburger W. TANDEM: integrating automated allele binning into genetics and genomics workflows. Bioinformatics. 2009:25:1982-3.

23. Glaubitz JC. CONVERT: a user-friendly program to reformat diploid genotypic data for commonly used population genetic software packages. Mol Ecol Notes. 2004;4:309-10.

24. Rousset F. GENEPOP'007: a complete re-implementation of the GENEPOP software for Windows and Linux. Mol Ecol Resour. 2008;8:103-6.

25. Excoffier $\mathrm{L}$, Lischer $\mathrm{HL}$. Arlequin suite ver 3.5: a new series of programs to perform population genetics analyses under Linux and Windows. Mol Ecol Resour. 2010;10:564-7.

26. Huho BJ, Ng'habi KR, Killeen GF, Nkwengulila G, Knols BGJ, Ferguson HM. Nature beats nurture: a case study of the physiological fitness of free living and laboratory-reared male Anopheles gambiae s. J Exp Biol. 2007;210:2939-47.

27. Charlesworth D, Charlesworth B. Inbreeding depression and its evolutionary consequences. Ann Rev Ecol Syst. 1987;18:237-68.

28. Falconer DS, Mackay TF, Frankham R. Introduction to quantitative genetics: trends in genetics. 4th ed. Harlow: Longman Frankel; 1996.

29. Lynch M, Walsh B. Genetics and analysis of quantitative traits. Sunderland: Sinauer Assoc. Inc.; 1998.

30. Hansson B, Westerberg L. On the correlation between heterozygotie and fitness in natural populations. Mol Ecol. 2002;11:2467-74.

31. Slate J, David P, Dodds KG, Veenvliet BA, Glass BC, Broad TE, McEwan JC. Understanding the relationship between the inbreeding coefficient and multilocus heterozygosity: theoretical expectations and empirical data. Heredity. 2004:93:255-65.

32. Chapman JR, Nakagawa S, Coltman DW, Slate J, Sheldon BC. A quantitative review of heterozygosity-fitness correlations in animal populations. Mol Ecol. 2009;18:2746-65.

33. Hendrichs J, Robinson AS, Cayol JP, Enkerlin W. Medfly areawide Sterile Insect Technique Programmes for prevention, suppression or eradication: the importance of mating behavior studies. Flo Entomol. 2002;85:1-13.

34. Calcagno GE, Manso F, Vilardi JC. Comparison of mating performance of medfly (Diptera: Tephritidae) genetic sexing and wild type strains: field cage and video recording experiments. Flo Entomol. 2002;85:41-50.

\section{Submit your next manuscript to BioMed Central and we will help you at every step:}

- We accept pre-submission inquiries

- Our selector tool helps you to find the most relevant journal

- We provide round the clock customer support

- Convenient online submission

- Thorough peer review

- Inclusion in PubMed and all major indexing services

- Maximum visibility for your research

Submit your manuscript at www.biomedcentral.com/submit
Ciomed Central 